\title{
Dynamic range extension of hybridization sensors
}

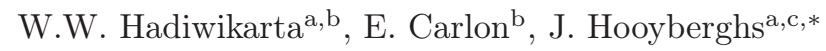 \\ ${ }^{a}$ Flemish Institute for Technological Research, VITO, Boeretang 200, B-2400 Mol, Belgium \\ ${ }^{b}$ Institute for Theoretical Physics, KULeuven, Celestijnenlaan 200D, B-3001 Leuven, Belgium \\ ${ }^{c}$ Theoretical Physics, Hasselt University, Campus Diepenbeek, Agoralaan - Building D, B-3590, Diepenbeek, Belgium
}

\begin{abstract}
In hybridization based nucleic acid sensors the stringency of hybridization poses a challenge to design and experiment. For a given set of experimental parameters the affinity window of probe-target interaction is always limited and vice versa for a given probe set design, changes in experimental conditions can easily bring some measurements out of detection range. In this paper we introduce and apply a strategy to extend this dynamic range for affinity sensors, sensors which measure the amount of hybridized molecules after equilibrium is reached. The method relies on concepts of additivity of nucleic acids hybridization free energies and on equilibrium isotherms. It consists in combining the measurements from probes with different length, by appropriately rescaling the measured signals. We test the validity of the approach on experiments and show that by combining probes with hybridizing regions of length 21,23 and 25 nucleotides we manage to extend the dynamic range of the intensity signals by a factor 25 . The presented concept is easy to extend, platform free and applies to any hybridization based affinity sensor.
\end{abstract}

Keywords: DNA, hybridization, stringency, dynamic range, thermodynamics

\section{Introduction}

For the detection of nucleic acid abundance or sequence variations there exists a large set of different methods which can be divided into two classes: hybridization based 5 techniques and enzyme based techniques. The latter are used in e.g. PCR and sequencing devices. The focus of this manuscript is on hybridization techniques which are appealing due to simplicity: a nucleic acid probe is designed to hybridize to the perfectly matching target molecule with

10 a high efficiency while having a low efficiency for targets containing sequence variations. The principle is open to many technical implementations and miniaturization for use in biosensor devices [1, 2, 3, 4, 5, 6]. However, hybridization has challenges on the side of probe design and dynamic range due to hybridization stringency conditions [7, 8, 9] which are not easy to optimize especially when parallelization is aimed for. The dynamic range of a sensor, i.e. the range in which the response signal is linear in concentration of the target, is an important concept and the control of it of high practical value for any sensor type. The presented work considers this issue for DNA affinity sensors, i.e. sensors which measure equilibrated signals without kinetic observations.

We address this issue with in mind the application DNA genotyping by single nucleotide polymorphism (SNP) detection. A SNP is a DNA sequence variation between

\footnotetext{
* Corresponding author

Email address: jef.hooyberghs@vito.be (J. Hooyberghs)
}

humans (or other members of the same species) consisting of a single nucleotide difference. It accounts for the majority of the genome variations between people and is functionally highly relevant. The detection of SNPs is important in many research and application domains like genome-wide associations studies, personalized molecular diagnostics and forensic identification [10, 11, 12, 13, 14, 15].

To detect SNPs on a given sequence by a hybridization technique one should use a sensor which contains the complementary probes of the possible target variants. In such a setup target molecules hybridize to different probes with different affinity: to their perfect complement, but also to probe sequences containing one or more mismatches. To make an optimal differentiation between them, they should all fall within the dynamic range of the detecting device. This is the region in which the measured signal $I$ is proportional to the target concentration: $I \propto c$. The intrinsic limitation, also in practice, is that a biosensor has a limited window of detection in which "too low" or "too high" signals are not exploitable.

In the current work we present a way to face this problem, we define the dynamic range of a hybridization affinity device and we introduce a concept by which the range can be extended by quantitatively combining readouts of probes with different length. We show an experimental implementation of the principle by means of a microarray experiment in which we use three different probe lengths

55 which results in the extension of the dynamic range by a factor of 25. The concept is however general and more 
probe lengths will result in larger extension factors.

\section{Materials and Methods}

\subsection{Sample preparation and hybridization experiment}

The target in the experiment is a single stranded $\mathrm{DNA}^{\mathbf{1 1 5}}$ (ssDNA) oligo which consists of a hybridization region and a labeling region. The former is the part of the oligo of interest which hybridizes to the microarray probes, its sequence is presented in Table 1. The latter is added for

65 labeling purposes and consists of poly A spacer together with a barcode sequence 5'-AAAAAAAAAATCACACAGGAAA CAGCTATGACGCCAG-3'.

The microarray experiments were performed using the commercially available Agilent platform and followed a

70 standard protocol with Agilent products, as described subsequently. Each hybridization mixture contains the target sequence at $10 p M$ together with a Cy3-labeled Barcode (Cy3-5'-AAAAACTGGCGTCATAGCTGTTtCCTGTGTGA-3') diluted in nuclease-free water to a final concentration of

${ }_{75} \quad 0.5 \mu \mathrm{M}$. The solution contains also $5 \mu \mathrm{l} 10 \times$ blocking agent and $25 \mu \mathrm{l} 2 \times$ GEx hybridization buffer HI-RPM. The hybridization mixture was centrifuged at $13000 \mathrm{rpm}$ for 1 minute and the microarray of the $8 \times 15 \mathrm{~K}$ custom Agilent slide was loaded with $40 \mu \mathrm{l}$ of the mixture. The hybridizaso tion occurred in an Agilent oven at $65^{\circ} \mathrm{C}$ for $17 \mathrm{~h}$ with rotor setting 10 and the washing was performed according to the instructions of the manufacturer. The array was scanned on an Agilent scanner (G2565BA) at $5 \mu \mathrm{m}$ resolution, high and low laser intensity and further processed using Agilent

${ }_{85}$ Feature Extraction Software (GE1 v5 95 Feb07) that performs automatic gridding, intensity measurement, background subtraction and quality checks. The choice for a barcoded indirect labeling design is not essential here, it was made to have a multipurpose assay without the need to label any target sequence.

\subsection{Probeset design}

In the microarray experiment a large number of different probes were used (in total 1836). The design of the probeset combines nucleotide variations and probe length 95 variations. The former is comparable to designs previously used to detect SNPs [16]. The probes carry up to two mismatches with respect to the target sequence. A few examples of sequences of this large probeset are shown in Table 1. Sequences (a-c) are perfectly matching to the tarbut they differ in lengths and overlap the hybridizing region with $L=25, L=23$ and $L=21$ nucleotides. (d-i) are examples of probes containing mismatches. Also these probes have three different lengths: $L=21,23$ and 25 . The mismatching nucleotides are underlined.

The constraint in designing the probe sequence is that the mimimal distance between two mismatches is of $4 \mathrm{nu}-$ cleotides and the mismatches have a minimal distance of 4 nucleotides to the ends of the hybridizing region (unless for L21 probes that have up to 3 nucleotides distance to
10 the ends of the hybridizing region). This follows the strategy discussed in [17]. As mismatches are sufficiently far apart their effect in the affinity should be considered as additive. By selecting them sufficiently far from the ends of the hybridizing region one can neglect end effects red (it is known that mismatches close to the end of the double helix are less destabilizing compared to mismatches in the bulk [17, 18, 19]).

\begin{tabular}{|c|c|}
\hline L & Hybridization region target sequence \\
\hline 25 & $5^{\prime}-\mathrm{CTTGCCTACGCCACCAGCTCCAACT-3^{ \prime }}$ \\
\hline $\mathbf{L}$ & Microarray probes sequence \\
\hline 25 & $5^{\prime}-\mathrm{AGTTGGAGCTGGTGGCGTAGGCAAG-3^{ \prime } ( a )}$ \\
\hline 23 & $5^{\prime}-\mathrm{GTTGGAGCTGGTGGCGTAGGCAA-3^{ \prime } ( b )}$ \\
\hline 21 & $5^{\prime}-$ TTGGAGCTGGTGGCGTAGGCA-3'(c) \\
\hline ... & $\ldots \ldots \ldots$ \\
\hline 25 & $5^{\prime}-\mathrm{AG} T T G G A G C T G G C G G C G T A G G C A A G-3^{\prime}(d)$ \\
\hline 23 & 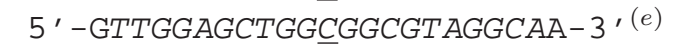 \\
\hline 21 & $5^{\prime}-T T G G A G C T G G \underline{C} G G C G T A G G C A-3^{\prime}(f)$ \\
\hline .. & $\ldots \ldots \ldots$ \\
\hline 25 & $5^{\prime}-\mathrm{AG} T T G G A G A T G G T G G T G T A G G C A A G-3^{\prime}(g)$ \\
\hline 23 & $5^{\prime}-\mathrm{GTTGGAGA} T$ TGTTGGTGTAGGCAA-3'(h) \\
\hline 21 & $5^{\prime}-$ TTGGAGATGGTGGTGTAGGCA-3'(i) \\
\hline$\ldots$ & \\
\hline
\end{tabular}

Table 1: Sequence of hybridization region of the target and of examples of probes immobilized on the surface of the microarray. The probes (a-c) are perfectly matching to the target sequence, however they differ in length (given in the left column). The probes (d-i) are sequences with mismatches.

\subsection{Langmuir isotherm and dynamic range}

When nucleic acid targets hybridize on immobilized surface probes its equilibrium state can be described by the Langmuir isotherm [20, 21, 22]. Assuming that the fraction of hybridized probes in a microarray spot is proportional to the measured signal intensity, for a DNA double helix in thermodynamic equilibrium, the isotherm can be written as

$$
I=\max \left(I_{0}, A \frac{c e^{-\Delta G / R T}}{1+c e^{-\Delta G / R T}}\right)
$$

where $I$ is the detected intensity signal, $I_{0}$ the lower detection limit of the readout, $A$ the maximum intensity when a spot is saturated by target molecules, $c$ the target concentration in solution, $R$ the ideal gas constant, $T$ the experimental temperature and $\Delta G$ is the hybridization free energy which determines the affinity of target-probe duplexes in a sequence dependent way. In hybridization experiments the temperature and target concentration are usually fixed, hence it is instructive to analyze the dependence of spot intensities on the free energy of the targetprobe duplex. Pane A of Figure 1 gives a visualization of equation (11) for realistic values of the physical parameters. 

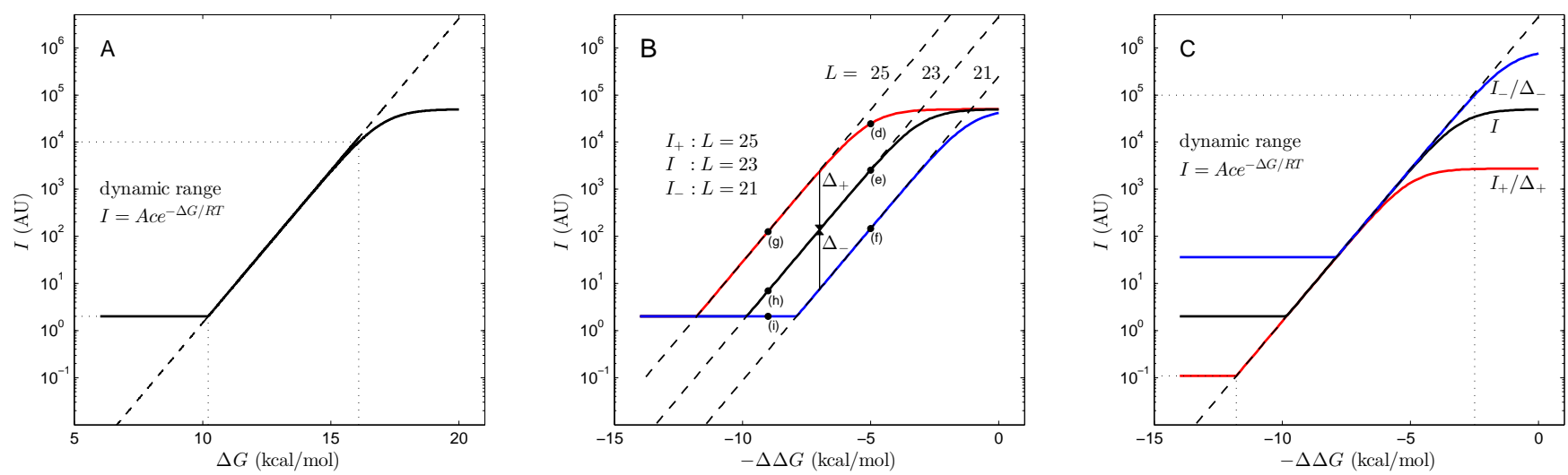

Figure 1: Series of plots to visualize the theory behind the dynamic range extension. Pane A: Solid line $=$ plot of intensities versus free energy of probe-target duplex according to the Langmuir isotherm. The physical parameters are: $A=5 \times 10^{4}, I_{0}=2, T=65^{\circ} \mathrm{C}, c=10 p M$. The dotted lines indicate the dynamic range, where the intensities follow closely the working regime (formula indicated) represented by the dashed line. Pane B: Similar plot but now versus free energy penalty $\Delta \Delta G$ and for the three probe lengths. $I_{+}, I, I_{-}$correspond to the intensities for probes of length 25,23,21 respectively. Two successive curves have an overlap in the dynamic range. Pane C: Rescaled plot of pane B. I.e. a plot of $I_{+} / \Delta_{+}, I, I_{-} / \Delta_{-}$. The axes of pane A and pane $\mathrm{C}$ are on the same scale, the increase of dynamic range can be evaluated from the dotted lines. Note that the intensities generated in pane $\mathrm{C}$ cover a range outside of the original range of detection of the sensor $\left(I_{0} \leq I \leq I_{0}+A\right)$.

From this equation one can read that when the target con-140 centration is very high or the duplex affinity very strong, i.e. $c e^{-\Delta G / R T} \gg 1$, the signal will hit the saturation limit thus $I \approx A$. If the concentration is very low or the affinity is very weak, i.e. $A c e^{-\Delta G / R T}<I_{0}$, the signal cannot drop below below the detection limit thus $I=I_{0}$. Thes45 inequalities

$$
I_{0}<A c e^{-\Delta G / R T} \ll A
$$

define the dynamic range of the hybridization sensor. Within this range a device is in its working regime and equation (1) can be approximated by

$$
I \approx A c e^{-\Delta G / R T}
$$

In Figure 1 this function is plotted as a dashed line. Within the dynamic range (2) the measured signal intensities are responsive in a uniform way to changes in target concentration or probe-target affinities. This range imposes a limited window of observation, which for our experiments can be read from pane A of Figure 1 roughly $I$ has a window of 3 orders of magnitude and $\Delta G$ a window of about $5 \mathrm{Kcal} / \mathrm{mol}$. In practice, it is a challenge to design probes in such a way that they all fall within the dynamic range. Therefore in our design we combine probes with different lengths to broaden the $\Delta G$ range and most importantly we propose a way to quantitatively combine them to extend the $I$ range.

\subsection{Extending the dynamic range}

The probes anchored in the microarray surface differ by length and sequence composition (see Table 1). The probe producing the highest signal intensity is sequence (a) in Table 1, i.e. the longest probe which is perfectly matching to the target sequence. This is the binding with the highest $-\Delta G$ (in what follows we will use the notation $\Delta G(a), \Delta G(b) \ldots$ to indicate the hybridization free energy for sequences (a), (b) ... of Table 1). According to the nearest-neighbor model [18] the hybridization free energy $\Delta G$ can be written as a sum of dinucleotide terms accounting for hydrogen bonding and stacking interactions. We exploit this additivity principle in the analysis of the microarray data. Consider as an example the sequences (a) and (d) in Table1 1 which differ only by a central nucleotide. Additivity implies that the difference $\Delta G(d)-\Delta G(a)$ depends only on the identities of the differing nucleotides and of the flanking ones. Therefore $\Delta G(d)-\Delta G(a)=$ $\Delta G(e)-\Delta G(b)=\Delta G(f)-\Delta G(c) \equiv \Delta \Delta G$, as these three pairs of sequences share the same local difference in nucleotides. Through this relation one defines a free energy penalty $\Delta \Delta G \equiv \Delta G(M M, L)-\Delta G(P M, L)$, where $\Delta G(P M, L)$ is the free energy of a perfectly matching probe with length $L$ (sequences (a), (b) or (c) in Table 1) and $\Delta G(M M, L)$ is the free energy of a mismatching probe with length L (sequences (d) to (i) in Table 1).

If we replot the data as a function of $\Delta \Delta G$ we got three intensity values $I_{+}, I$ and $I_{-}$which correspond to probe length $\mathrm{L}=25,23$ and 21 (see Fig. 1(B)). Hence, in our design every sequence variation is represented by a single free energy penalty $\Delta \Delta G$ and by three intensity measurements, one for each probe length. Figure 1(B) is key to the idea of dynamic range extension. The dynamic range (defined by (2)) of each probe length has a different $\Delta \Delta G$ window. Depending on the free energy penalty of the mismatches probes of different lengths fall within the dynamic range. In the example in Fig. 1(B) the sequences (d) and (i) fall outside the dynamic range. The idea is to use the shorter (or longer) probes for the data in the high (or low) intensity range.

Consider now two probe sequences sharing the same mismatch and which both fall within the dynamic range of the device. For instance sequences (e) and (f) of Table 1 . 
As these fall within the dynamic range one can use Eq. (3) and write for the ratio of the two intensities:

$$
\frac{I(f)}{I(e)}=\exp [(\Delta G(e)-\Delta G(f)) / R T]
$$

We can now again exploit the additivity principle and note that for the sequence in Table 1 we have

$$
\begin{aligned}
\Delta G(d)-\Delta G(e) & =\Delta G(g)-\Delta G(h) \equiv \delta_{+} \\
\Delta G(e)-\Delta G(f) & =\Delta G(h)-\Delta G(i) \equiv \delta_{-}
\end{aligned}
$$

because the corresponding probes only differ in the fixed boundary nucleotides. Next denote

$$
\begin{aligned}
& \Delta_{-}=e^{-\delta_{-} / R T} \\
& \Delta_{+}=e^{\delta_{+} / R T} .
\end{aligned}
$$

Within the dynamic range, the form of the Langmuir isotherm implies that $I_{+}=I / \Delta_{+}$and $I_{-}=I / \Delta_{-}$. In other words, the three curves of Figure 1(B) are parallel to each other ithin the dynamic range and their "vertical distances" are given by the scaling factors $\Delta_{+}$. Hence we can shift $I_{+}$and $I_{-}$vertically to map them on $I$, the result is shown in Figure 1(C). By combining a shorter and a longer probe sequence in our measurement and by correctly scaling the ities, we increased the dynamic range of $I$ by a factor $\Delta_{+} / \Delta_{-}$. In the next section we show how this increase is achieved in the analysis of experimental data.

Note that two main thermodynamical properties fol 210 lowing from additivity were crucial in the argument: (i) he free energy penalty of a probe mismatch $(\Delta \Delta G)$ is independent on the probe length $L$ and (ii) the free energy penalty of probe length is independent of mismatches.

\section{Results and discussion}

\subsection{Determination of scaling factors}

In this section we show how the scaling factors $\Delta_{ \pm_{220}}$ can be extracted from experimental data, in this case the microarray data presented in the Materials and Methods section. A practical and robust way to determine the scaling factors from experiments is to plot the spot intensities plot is shown in Figure 2.

In this approach we avoid the use of free energy $\Delta G$ (or $\Delta \Delta G$ ). This is important since free energy cannot be extracted directly from the experiment itself and needs to modeled. Although free energy parameters obtained from the analysis of a large number of microarray data are available in the literature, these modeled quantities are less accurate than experimental intensities. Plotting intensities vs. intensities as done in Figure 2 bypasses this problem. In this plot the central branch is the diagonal $I$ vs. $I$. The two other branches $I_{+}$and $I_{-}$are in part parallel to the diagonal. The intensities are bounded by ${ }^{235}$ the minimal background level $I_{0}$ and the maximal intensity

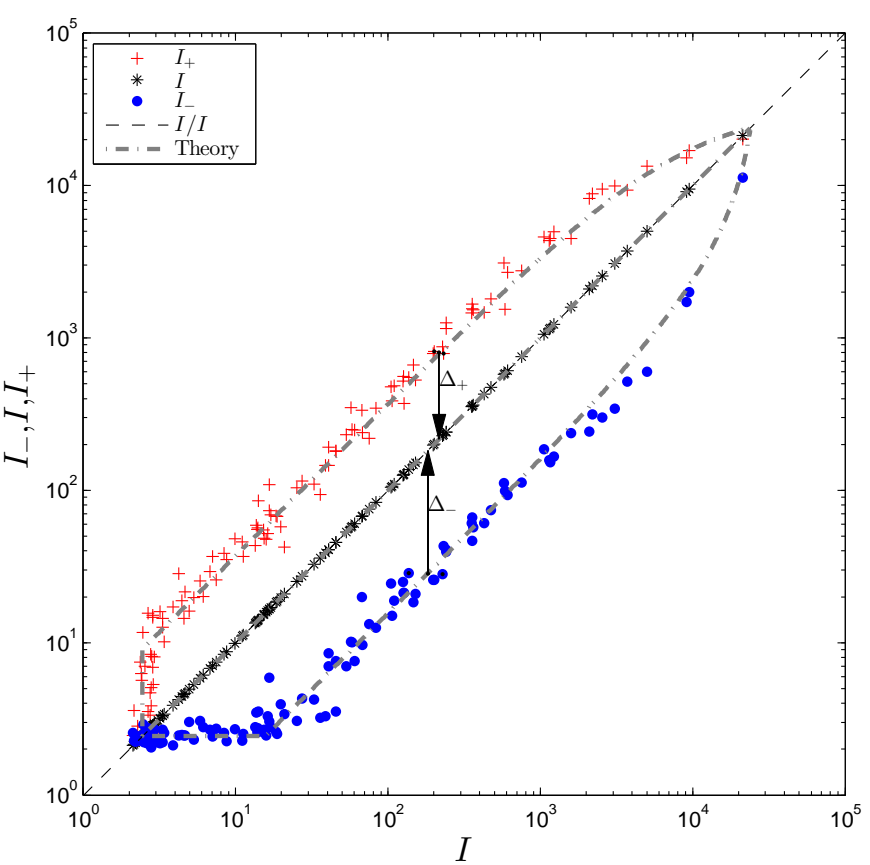

Figure 2: Experimental data: spot intensities $I_{-}, I$, and $I_{+}$(probe lengths $L=21,23,25$ ) against $I$. From this plot the scaling factors $\Delta_{-}$and $\Delta_{+}$are extracted. The dotted lines on top of experimental data are the theoretical Langmuir curves, from Figure 1 using the fitted $\Delta_{ \pm}$values.

level $A$. The deviations of the two side branches from being parallel to the diagonal is where one or more of the intensities deviate from the linear regime of Eq. (21).

The experimental data of $I_{+}(L=25$ probes $)$ and $I_{-}$ ( $L=21$ probes $)$ vs. I ( $L=23$ probes), follow closely the expected behavior from thermodynamics (dotted lines, gion, the data is clean and linear, which is an experimental proof that free energy penalty of probe length is independent from mismatches. This observation makes a solid dynamic range extension possible. We determined the region where the $I_{+}$and $I_{-}$branch are parallel to the diagonal and estimated the scale factors $\Delta_{+}$and $\Delta_{-}$ from the ratios $I_{+} / I$ and $I / I_{-}$averaged over corresponding pairs of probes. From the analysis of the experimental data we find $\Delta_{+}=3.7$ and $\Delta_{-}=0.15$ which corresponds to the free energies differences $\delta_{-}=1.27 \mathrm{Kcal} / \mathrm{mol}$ and $\delta_{+}=0.88 \mathrm{Kcal} / \mathrm{mol}$ using equation (6). Note the asymmetry $\left(\delta_{+} \neq \delta_{-}\right)$which is to be expected since these values are related to the specific free energy of the extra boundary nucleotides (see Table 1). Based on our probe sequences this free energy is expected to be different for the different lengths [18].

\subsection{Rescaling of intensity data}

To finalize the analysis we rescale the intensity data by $\Delta_{ \pm}$. In order to show the result we need to produce a plot versus $\Delta \Delta G$, like pane $\mathrm{C}$ of Figure 1 Hereto we have to calculate, for each probe, the free energy penalties 

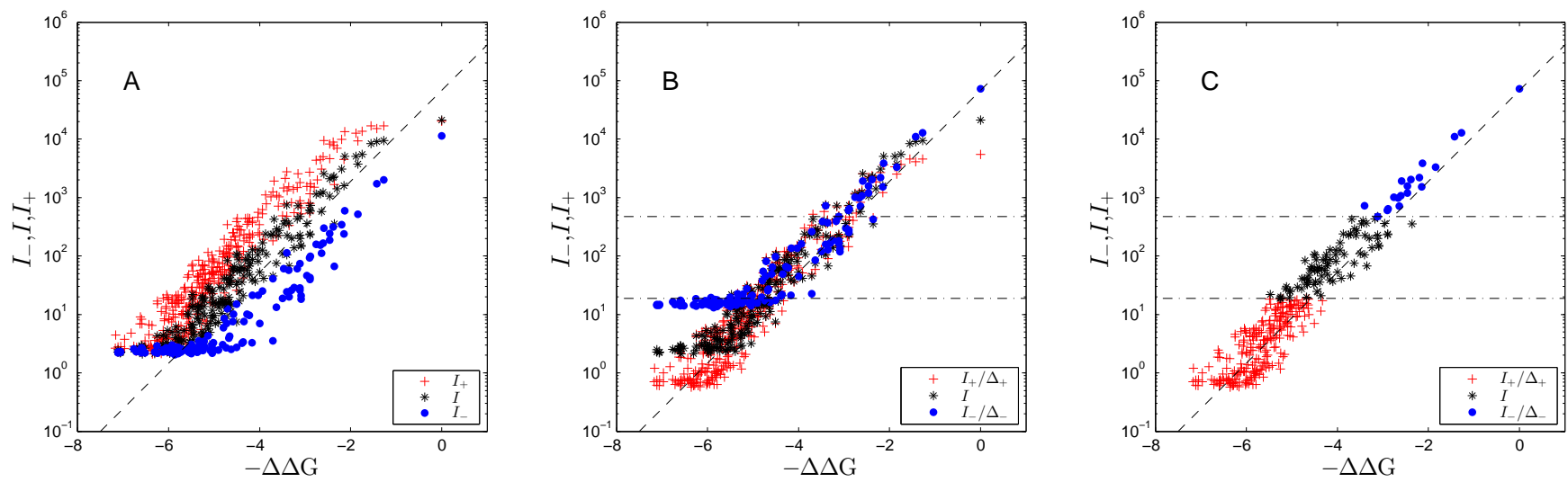

Figure 3: Plots of experimental data vs free energy $\Delta \Delta G$. Pane A: plot in the same way like Pane B of Figure 1 but using experimental data. For low intensities, the signals are flat and for high intensities, $I_{+}(L=25)$ and $I(L=23)$ signals are saturated. Pane B: the rescaled intensities $I / \Delta_{+}$and $I / \Delta_{-}$are shown. We split the data into three regions: low-, mid- and high intensity, separated by the two dashed lines. Pane C: the signals are selected. For the low intensity region, signals from $I / \Delta_{+}$are used. $I$ signals are used in the mid intensity region and $I / \Delta_{-}$signals are for the high intensity region. 8, 9]. In this paper we address the issue of extending the using signal $I_{+}$( $L=25$ probes) for the low intensities, $I_{-}$ ( $L=21$ probes) for high intensities and $I$ ( $L=23$ probes) for middle intensities. The result is shown in Pane C. I ${ }^{\beta}{ }^{95}$ we compare Pane $\mathrm{C}$ and Pane $\mathrm{A}$, the extension of the dynamic range of the device is clear and can be quantified by the factor $\Delta_{+} / \Delta_{-} \approx 25$.

\section{Discussion and conclusions} poses a challenge to the experimental dynamic range $7_{30}$

dynamic range of an hybridization affinity sensor, a sensor which measures the amount of hybridized molecules after equilibrium is reached. This is performed by a design in which different probe lengths are used for each probe sequence and most importantly by a concept to quantitatively combine experimental intensities of the probes with different length. The method relies on the principle of free energy additivity, where the data from probes of different lengths are rescaled using principles from equilibrium thermodynamics. Specifically in our experiments, the combined and extended data of Pane $\mathrm{C}$ do show linear behavior over (nearly) the whole free energy range and the dynamic range is extended by a factor 25 . The gained factor is related to the current experimental design. In principle this dynamic range can be extended further if data from longer and shorter probes are used and rescaled using the same principles as illustrated in this paper. Hence, the concept provides a flexible way to extend the dynamic range and face the stringency of a hybridization affinity device.

\section{Acknowledgement}

We thank An Jacobs for expert technical assistance. Conflict of interest: patent application filed.

\section{References}

[1] Cornett, E. M., O'Steen, M. R., and Kolpashchikov, D. M. (2013) PLoS ONE 8, e55919.

[2] Janse, I., Bok, J. M., Hamidjaja, R. A., Hodemaekers, H. M., and van Rotterdam, B. J. (2012) PLoS ONE 7, e31958.

[3] Vanden Bon, N., van Grinsven, B., Murib, M. S., Yeap, W. S., Haenen, K., De Ceuninck, W., Wagner, P., Ameloot, M., Vermeeren, V., and Michiels, L. (2014) Int. J. Nanomedicine 9, 1629-1640.

[4] Tomlinson, J., Harrison, C., Boonham, N., Goodchild, S. A., and Weller, S. A. (2014) BMC Res. Notes 7, 251.

[5] Cherstvy, A. G. (2013) Biosens. Bioelectron. 46, 162-170. 
[6] Mahadhy, A., Ståhl-Wernersson, E., Mattiasson, B., and Hedström, M. (2014) Biotech. Reports 3, 42-48.

[7] Yang, H., Wanner, I. B., Roper, S. D., and Chaudhari, N. (1999) J. Histochem. Cytochem. 47, 431-446.

[8] Sharov, V., Kwong, K. Y., Frank, B., Chen, E., Hasseman, J., Gaspard, R., Yu, Y., Yang, I., and Quackenbush, J. (2004) BMC Biotechnol. 4, 3.

[9] Sassolas, A., Leca-Bouvier, B. D., and Blum, L. J. (2007) Chem. Rev. 108, 109-139.

[10] Gresham, D., Dunham, M. J., and Botstein, D. (2008) Nat. Rev. Genet. 9, 291-302.

[11] McCarroll, S. A. (2008) Hum. Mol. Genet. 17, R135-R142.

[12] Holbrook, J., Parker, J., Gallagher, K., Halsey, W., Hughes, A., Weigman, V., Lebowitz, P., and Kumar, R. (2011) J. Transl. Med. 9, 119.

[13] Blakemore, A. I. F., and Froguel, P. (2010) Ann. N. Y. Acad. Sci. 1214, 180-189.

[14] Kling, D., Welander, J., Tillmar, A., Skare, Ø., Egeland, T., and Holmlund, G. (2012) Forensic Sci. Int.-Genet. 6, 322-329.

[15] Freire-Aradas, A., Fondevila, M., Kriegel, A. K., Phillips, C., Gill, P., Prieto, L., Schneider, P. M., Carracedo, Á., and Lareu, M. V. (2012) Forensic Sci. Int.-Genet. 6, 341-349.

[16] Hooyberghs, J., and Carlon, E. (2010) Biosens. Bioelectron. 26, $1692-1695$.

[17] Hadiwikarta, W. W., Walter, J.-C., Hooyberghs, J., and Carlon, E. (2012) Nucleic Acids Res. 40, e138.

[18] SantaLucia, Jr., J., and Hicks, D. (2004) Annu. Rev. Biophys. Biomol. Struct. 33, 415-440.

[19] Letowski, J., Brousseau, R., and Masson, L. (2004) J. Microbiol. Methods 57, 269-278.

[20] Carlon, E., and Heim, T. (2006) Physica A. 362, 433-449.

[21] Held, G. A., Grinstein, G., and Tu, Y. (2003) Proc. Natl. Acad. Sci. U. S. A. $100,7575-7580$.

[22] Hooyberghs, J., Van Hummelen, P., and Carlon, E. (2009) $\mathrm{Nu}$ cleic Acids Res. 37, e53.

[23] Bloomfield, V. A., Crothers, D. M., and Tinoco, I. University Science Books, 2000. 